\title{
Et si la croissance n'était pas au rendez-vous ?
}

\author{
Michel Boisvert \\ Universit de Montr al
}

\section{Introduction}

Toute région dispose de ressources, matérielles et humaines, qui ne demandent qu'à être exploitées. La mise en valeur des ressources disponibles dans une région, sur laquelle s'appuient notamment tous les courants guidés par le concept de développement endogène, est donc la réponse facile à cette question. À vrai dire, cette démarche engage non seulement les personnes qui habitent une région, les premiers bénéficiaires des actions entreprises, mais, en raison des liaisons interrégionales, elle engage aussi celles qui résident dans les régions avec lesquelles sont entretenus des liens étroits. Ceux-ci peuvent prendre la forme d'échanges commerciaux, de flux de capitaux ou d'investissements, voire d'informations nécessaires à la prise de décision dans les entreprises. Cette vision inspire notamment l'approche du développement en filières de production et a produit des concepts puissants comme les pôles de croissance et les pôles de développement, abondamment utilisés il y a une trentaine d'années par les professeurs Higgins, Martin et Raynauld de l'Université de Montréal dans une proposition de stratégie économique pour le Québec. Ce document a été fort critiqué à l'époque «en région » et jeté dans l'oubli, malgré d'incontestables qualités.

Mais la question posée pour ce numéro spécial de la revue cache en fait des sous-questions beaucoup plus complexes, dans la mesure où les efforts de développement de tous les acteurs concernés impliquent des choix qui d'une part doivent s'harmoniser les uns aux autres et qui, d'autre part, doivent respecter certaines contraintes liées aux ressources financières à la disposition des acteurs publics. Nous chercherons donc à répondre d'abord à la question suivante : «Une stratégie de développement peut-elle s'accommoder de ressources inutilisées et de travailleurs qui émigrent en grand nombre? » Puis nous nous intéresserons aux contraintes budgétaires de l'État, en nous demandant si l'équité dans l'allocation des aides gouvernementales et des équipements publics ne favorise pas la concentration dans les grands centres au détriment des régions, dans lesquelles la population est plutôt dispersée. Les réponses à ces deux interrogations devraient nous aider enfin à répondre à une dernière question, plus globale et ouverte : "Quelle est la place des régions non métropolitaines à l'ère des NTIC et de la mondialisation des marchés?»

\section{Décroissance $=$ Développement ?}

On distingue souvent la croissance et le développement économiques, du moins sur le plan théorique, en associant le premier processus à l'évolution d'indicateurs de performance, essentiellement des extrants (volume de production, nombre de salariés, pouvoir d'achat), dans des conditions connues de technologie, de comportement et d'environnement institutionnel. Le processus de développement est de son côté appréhendé en accordant plus d'importance aux intrants (niveau de qualification de la main-d'œuvre, nombre de kilomètres d'autoroutes ou de chemins de fer par 1000 habitants, taux de mortalité infantile), dans des conditions évolutives de technologie, de comportement et d'environnement institutionnel. C'est ainsi qu'on refusera de conclure au développement économique d'une région sur la seule base d'indicateurs de croissance. Le plus bel exemple d'un tel refus est sans doute l'idéologie du développement durable, qui condamne la croissance lorsqu'elle s'accompagne de la destruction de l'environnement et ne se soucie aucunement de l'évolution du capital 
humain.

Mais alors, si la croissance n'est pas garante du développement, peut-on imaginer que la décroissance s'inscrive dans un processus de développement? Assurément, mais pas n'importe comment. Cette décroissance peut prendre la forme de ressources inemployées. Tel est le cas lorsque les forêts ou les sols arables sont soustraits à l'exploitation commerciale ou lorsque le potentiel minier demeure inexploité. C'est aussi le cas lorsque des travailleurs sont au chômage ou choisissent de se retirer volontairement de la population active. Comment y voir un apport au développement régional ? Ces ressources peuvent profiter de cette soustraction au processus de production pour se régénérer; c'est bien l'une des explications de la jachère évoquée plus haut. Les chômeurs et inactifs peuvent aussi mettre à profit cette situation en améliorant leur productivité, grâce par exemple à des programmes de formation professionnelle dans leur domaine actuel d'activité, voire dans un tout autre domaine. En d'autres mots, un volume de production est soustrait pour une durée limitée, dans la perspective d'une augmentation future, au moment où les ressources régénérées seront à nouveau mises en service.

Une autre explication, plus courante, découle du concept de coût d'option. Ce dernier mesure les bénéfices nets associés à l'affectation d'une ressource à un autre usage que l'usage courant. On peut par exemple freiner l'exploitation forestière de manière à faciliter le développement de l'activité récréotouristique dans une région. On peut aussi, avec l'accord de la Commission de protection du territoire agricole, réaffecter à une fonction urbaine, par exemple résidentielle, des terres jusque-là consacrées à l'activité agricole. Sur le plan de la croissance de la production, le premier exemple implique vraisemblablement une perte de valeur de production, compensée par une amélioration de la qualité environnementale. Dans le second exemple, il $\mathrm{y}$ a perte sèche de valeur ajoutée, à moins d'un transfert d'activité sur de nouvelles terres mises en production.

Cette réaffectation peut aussi s'appliquer aux travailleurs lorsque ceux-ci décident de changer d'entreprise ou même de secteur d'activité voire de déménager dans une autre région afin d'améliorer leur employabilité ou leur niveau de salaire. Sur le plan individuel, une telle décision est tout à fait compréhensible, mais elle prend à l'échelle collective une toute autre signification. Car la décroissance signifie entre autres des marchés immobiliers qui s'effondrent et des équipements, privés et publics, qui n'arrivent plus à se renouveler, quand ils réussissent à survivre. Il n'est qu'à penser aux installations aéroportuaires et aux services qu'ils assurent, ou encore aux équipements hospitaliers et aux bureaux de poste. Une stratégie de développement régional, élaborée et mise en œuvre par tous les acteurs concernés - et ils comprennent des non-résidents - ne peut donc ignorer ces contrecoups qui appellent des mécanismes de compensation et l'identification de niveaux-planchers pour les services à assurer et ce, non seulement par souci de solidarité sociale mais aussi pour des raisons de clairvoyance. Car comment encourager la relance éventuelle si certaines conditions de cette relance viennent à disparaître ? Cette approche, pour réussir, impose donc à l'État, en partenariat avec la société civile, un rôle proactif et des engagements fermes.

Une stratégie de développement régional, élaborée et mise en œuvre par tous les acteurs concernés - et ils comprennent des non-résidents - ne peut donc ignorer ces contrecoups qui appellent des mécanismes de compensation et l'identification de niveauxplanchers pour les services à assurer et ce, non seulement par souci de solidarité sociale mais aussi pour des raisons de clairvoyance. Car comment encourager la relance éventuelle si certaines conditions de cette relance viennent à disparaître ? Cette approche, pour réussir, impose donc à l'État, en partenariat avec la société civile, un rôle proactif et des engagements fermes.

\section{Rendement régional variable ?}

Le développement passe par des investissements publics, sous la forme de nouveaux équipements ou d'aides gouvernementales. L'État, que ce soit à l'échelle fédérale ou provinciale, est tout à fait justifié de viser d'abord un objectif d'efficience, c'est-à-dire de chercher à maximiser les avantages nets pour la 
société dans l'allocation de ses ressources. Rappelons ici à ceux qui n'y verraient qu'un credo néolibéral que l'analyse bénéfices - coûts exige de prendre en compte les coûts environnementaux ainsi que les coûts et avantages sociaux, ce qui signifie par exemple qu'il faille considérer non seulement comme coûts mais aussi comme bénéfices les salaires versés à des travailleurs qui, en l'absence de tels investissements publics, demeureraient au chômage. Mais, en intégrant la pratique des enveloppes budgétaires sectorielles, qui mène à rechercher les rendements les plus élevés plutôt que les bénéfices nets actualisés les plus grands, rien ne dit que ces rendements, même après introduction des dimensions environnementales et sociales, seront uniformes à travers toutes les régions. Tel n'est pas le cas dans le secteur privé. Pourquoi donc en serait-il autrement dans le secteur public, malgré une comptabilité plus complexe ? Qui peut croire que les bénéfices découlant d'investissements dans la construction d'aéroports, de campus universitaires ou même d'hôpitaux spécialisés seront les mêmes peu importe leur localisation ? Il faut donc s'attendre à ce que certaines régions reçoivent plus d'investissements publics que d'autres, relativement à leur part de la population ou de la production (PIB). Ceci implique la présence de rythmes de croissance différents d'une région à l'autre dans la mesure où ces investissements publics accompagnent à leur façon les investissements privés et qu'ils annoncent tous deux l'évolution attendue de l'activité de production.

On peut même s'attendre, au risque de simplifier, à ce qu'en général, les rendements soient plus élevés dans les régions métropolitaines par opposition aux régions où la population est dispersée, et l'éventail des activités et des qualifications de la main-d'œuvre plus réduit. En effet, les coûts de transport y seront moindres et, en raison de la concentration d'expertises plus pointues, les bénéfices attendus en matière de productivité seront accrus.

Mais l'intervention publique ne saurait être guidée par les seules considérations d'efficience. Les aides gouvernementales, sous forme de subventions, ne doiventelles pas faire place à l'équité ? Rappelons tout de même que les aides financières sont dans certaines circonstances un simple véhicule différent pour permettre à l'État d'intervenir : au lieu de prendre lui-même les décisions en matière de production ou de consommation, il laisse les acteurs sociaux prendre leurs déci- sions et se donne le pouvoir d'orienter ces choix par des incitatifs. Il sera donc parfois guidé principalement par l'enjeu de l'efficience déjà évoqué, mais à d'autres moments, il est vrai, par sa préoccupation de justice sociale. Et celle-ci se doit d'englober une perspective régionale, puisque la dimension collective s'exprime souvent dans un contexte territorial. Nous voilà ramenés aux deux dispositions mentionnées plus haut, soit la nécessité d'en atténuer les impacts et celle d'assurer les conditions minimales de viabilité sociale et économique.

L'État sera parfois guidé principalement par l'enjeu de l'efficience, mais à d'autres moments, il est vrai, par sa préoccupation de justice sociale. Et celle-ci se doit d'englober une perspective régionale, puisque la dimension collective s'exprime souvent dans un contexte territorial. Nous voilà ramenés aux deux dispositions mentionnées plus haut, soit la nécessité d'en atténuer les impacts et celle d'assurer les conditions minimales de viabilité sociale et économique.

Ce ne sont là que des amortisseurs, pourrait-on rétorquer, le moteur du développement régional demeurant sous le contrôle du secteur privé. C'est pourtant là un simple constat d'une situation qui ne semble pas près de changer. Mais alors, ces disparités interrégionales dans les conditions d'opération économique ne conduiront-elles pas, via une spirale du sous-développement, à des disparités chroniques dans les conditions de vie ? L'actualisation, prévue dans les analyses avantages - coûts, doit tenir compte de ces risques de détérioration, et il faut admettre qu'on ne le fait pas. Même alors, des écarts subsisteront probablement, que chaque individu aura à décider de supporter ou non. On sait par exemple que les Québécois, même parfaitement bilingues, sont prêts à tolérer un pouvoir d'achat plus faible pour demeurer au Québec plutôt que de s'expatrier ailleurs au Canada. Une partie de cette force d'inertie correspond à un calcul économique (meilleure information sur le marché du travail et opportunités d'avancement plus grandes 
grâce à un réseau plus étendu de contacts), et une autre partie à des considérations sociopolitiques, à commencer par les affinités culturelles. Comme en beaucoup d'autres sphères de l'analyse régionale, cette situation peut être transposée à une autre échelle, en l'adaptant en l'occurrence aux rapports entre Montréal et les autres régions du Québec.

\section{Les régions-ressources et les parcs de haute technologie}

Le ralentissement dans la croissance des régions métropolitaines des pays industrialisés à partir des années 1980 a fait croire à un retour de balancier, c'està-dire à un renouveau des régions périphériques. Un examen plus attentif a montré que la croissance s'était déplacée à la périphérie de ces espaces métropolitains et non dans les régions excentriques, nous obligeant à reconnaître la métapolisation, concept proposé par François Ascher. L'impulsion des nouvelles technologies de l'information et de la communication (NTIC) par exemple a eu deux effets. D'abord, celui de susciter la création d'une multitude d'entreprises vouées à la conception et à l'adaptation de ces nouveaux produits, et qui ont choisi massivement une localisation métapolitaine, comme en témoignent notamment ces parcs technoscientifiques qui ponctuent désormais le paysage urbain. L'impulsion des NTIC a aussi encouragé, sur le plan de ses applications, la mondialisation des marchés, moins dans le secteur primaire ou les industries de transformation (biens intermédiaires) que dans les industries de fabrication (biens finis), parce que l'internationalisation était dans le premier cas déjà acquise et que de toute façon, la mobilité des installations y est beaucoup plus restreinte. La croissance de la nouvelle économie, quand croissance il y a, est donc à l'avantage des régions métropolitaines étendues. $\mathrm{Au}$ Québec, cette région dont le pivot est Montréal forme en gros un triangle dont les sommets sont Ottawa -
11, Québec et Sherbrooke.

Et si la croissance n'était pas au rendez-vous ? Une fois vérifié que les décideurs qui choisissent de quitter la région (travailleurs qui émigrent, gouvernements qui refusent d'investir, entreprises qui recyclent leurs profits ailleurs) appuient leur décision sur des informations complètes et irréprochables, il faudra construire avec l'État les amortisseurs (mécanismes compensatoires) et les butées (services minima) qui sont la marque d'une société responsable.

Quel est le sort des régions excentriques ? Le développement de leurs ressources naturelles et de leurs ressources humaines est un défi immense, qui passe aussi par les nouvelles technologies, non seulement dans le secteur de l'information (qu'on pense à la géomatique) mais également dans des secteurs comme les biotechnologies ou le design industriel. Et ces régions ne sont pas condamnées à jouer un simple rôle de consommateur de ces nouvelles techniques, car leur conception même demande de faire appel à un réseau de centres technologiques. Il reste que le gros des effectifs se situera plutôt en région centrale. Et si la croissance n'était pas au rendez-vous ? Une fois vérifié que les décideurs qui choisissent de quitter la région (travailleurs qui émigrent, gouvernements qui refusent d'investir, entreprises qui recyclent leurs profits ailleurs) appuient leur décision sur des informations complètes et irréprochables, il faudra construire avec l'État les amortisseurs (mécanismes compensatoires) et les butées (services minima) qui sont la 